\title{
MET and MST1R as prognostic factors for classical Hodgkin's lymphoma
}

\author{
Young Wha Koh ${ }^{1}$, Chansik Park ${ }^{1}$, Dok Hyun Yoon ${ }^{2}$, Cheolwon Suh² and Jooryung Huh ${ }^{1}$ \\ ${ }^{1}$ Department of Pathology, University of Ulsan College of Medicine, Asan Medical Center, Seoul, South Korea \\ and ${ }^{2}$ Department of Oncology, University of Ulsan College of Medicine, Asan Medical Center, Seoul, South \\ Korea
}

\begin{abstract}
MST1R (RON) and MET are receptor tyrosine kinase gene family members that form a noncovalent complex on the cell surface, a critical step in tumor progression. A recent study suggested a prognostic role of MET expression in Hodgkin/Reed-Sternberg (HRS) cells in classical Hodgkin's lymphoma (cHL). The purpose of this study was to examine the prognostic significance of MET and MST1R expression in $\mathrm{cHL}$. The prognostic impact of MET and MST1R was examined in 100 patients with $\mathrm{cHL}$ (median age: 32 years) by immunohistochemistry and mRNA in situ hybridization. The median follow-up time was 95 months (interquartile range: 42-126 months). MET or MST1R protein expression was associated with high MET or MST1R mRNA expression, respectively. Thirty-eight patients (38\%) expressed MET protein in HRS cell, which was associated with better overall survival $(P=0.004)$. Twenty-six patients $(26 \%)$ expressed MST1R protein, which was associated with better overall survival $(P=0.022)$ and event-free survival $(P=0.021)$. Multivariate analysis identified MET protein as an independent prognostic factor for overall survival and MST1R protein as an independent prognostic factor for event-free survival. Subgroup analysis according to Ann Arbor stage showed that expressions of MET and MST1R protein have prognostic impact in the advanced stage only. In particular, coexpression of MST1R and MET protein was associated with a better survival outcome than MET or MST1R expression alone or no expression. This study suggests that MET and MST1R are independent prognostic factors in classical $\mathrm{cHL}$, and may allow the identification of a subgroup of $\mathrm{cHL}$ patients who require more intensive therapy.

Modern Pathology (2013) 26, 1172-1182; doi:10.1038/modpathol.2013.64; published online 5 April 2013
\end{abstract}

Keywords: Hodgkin's lymphoma; MET; mRNA; MST1R; prognosis

Classical Hodgkin lymphoma (cHL) is characterized by disruption of the normal lymph node architecture by the minority of malignant Hodgkin/ReedSternberg (HRS) cells. HRS cells are found in a background of bystander reactive cells composed of $\mathrm{T}$ and $\mathrm{B}$ lymphocytes and other cell types, including macrophages, eosinophils, basophils, and plasma cells. Deregulated signaling pathways, ${ }^{1,2}$ EpsteinBarr virus (EBV) infection, ${ }^{3-5}$ and an inflammatory microenvironment ${ }^{6-8}$ contribute to the survival and proliferation of HRS cells.

Despite important advances in the treatment of cHL, 5 to $10 \%$ of patients are resistant to initial therapy and 10 to $30 \%$ will relapse after initial remission. ${ }^{9}$ The standard stratification system for survival in patients with cHL is the International

Correspondence: Professor J Huh, MD, Department of Pathology, University of Ulsan College of Medicine, Asan Medical Center, 88, Olympic-ro 43-gil, Seoul 138-736, South Korea.

E-mail: jrhuh@amc.seoul.kr

Received 26 June 2012; revised 6 November 2012; accepted 10 January 2013; published online 5 April 2013
Prognostic Score (IPS), which is limited to patients with advanced-stage disease. ${ }^{10}$ Increased hematologic toxicity and secondary malignancies are major problems in cHL. Several clinical, biological, and laboratory prognostic factors have been examined for their prognostic utility. Although some prognostic markers have been reported, including early interim positron emission tomography, ${ }^{11}$ tumorassociated macrophages, ${ }^{6,8}$ and EBV status, ${ }^{5,12}$ their predictive clinical use in patients with $\mathrm{cHL}$ is still unclear.

The HGF/MET signaling pathway, which is a subfamily of receptor tyrosine kinases (RTKs), has drawn special attention to the association among tumor cell proliferation, survival, invasion, and metastasis. ${ }^{13,14}$ Deregulation and the consequent aberrant signaling of MET are caused by different mechanisms, including gene amplification, overexpression of activating mutations, and increased autocrine or paracrine ligand-mediated stimulation. These events can play important roles in the pathogenesis of cancer. ${ }^{15}$ Recent studies reveal that MET is overexpressed in gastric, ${ }^{16}$ colon, ${ }^{17}$ breast, ${ }^{18}$ 
ovary, ${ }^{19}$ kidney, ${ }^{20}$ lung, ${ }^{21}$ and thyroid carcinomas. ${ }^{22}$ Downregulation of MET expression by an antagonistic anti-MET antibody or small interfering RNA inhibits cellular proliferation and motility in lung cancer $^{23}$ and medullary thyroid cancer. ${ }^{24}$ Previous studies also report that MET/HGF has prognostic significance in many malignant tumors. ${ }^{19,20,25}$

MST1R (RON), an RTK with homology to MET, is involved in tumor progression and metastasis. ${ }^{26,27}$ MST1R is overexpressed in human epithelial malignancies. ${ }^{28,29}$ The expressions of MET and MST1R have been detected in HRS cells in patients with cHL. ${ }^{30,31}$ Recently, MET expression was reported to be a prognostic factor for clinical outcomes in $\mathrm{CHL}^{32}$ Although a relationship between MST1R and MET is observed in several malignancies, ${ }^{33,34}$ no study has examined the prognostic significance of MET and MST1R in cHL patients. Thus, the aim of the present study was to determine the clinical significance of MET/MST1R protein and mRNA expression in patients with cHL.

\section{Materials and methods}

\section{Patients}

The present report involved a retrospective study of 100 consecutive patients with cHL diagnosed at Asan Medical Center between 1990 and 2009. All of the patients met the following criteria: pathologically confirmed cHL; no previous treatment; no previous history of malignancy; and treatment with combination chemotherapy, with or without radiation treatment. Paraffin-embedded tumor tissues and follow-up data were available for all included patients.

Clinical information, including age, gender, Ann Arbor stage, presence or absence of B symptoms, IPS, serum lactate dehydrogenase (LDH) level, treatment regimen, and survival data, were obtained from the medical records. The median follow-up time for surviving patients was 95 months (interquartile range: 42-126 months). Response criteria were based on standard guidelines. Routine followup imaging analyses were performed every 3 months for the first 2 years, every 6 months for the next 3 years, and then annually (or whenever clinically indicated) thereafter.

\section{Histopathological Analysis and Immunohistochemistry}

The histological data of all patients were reviewed by three pathologists (JH and YWK). Histological subtype was classified according to the World Health Organization (WHO) criteria as follows: nodular sclerosis (NS), lymphocyte-rich (LR), mixed cellularity (MC), lymphocyte-depleted (LD), or not otherwise specified cHL.
A representative tumor section paraffin block (donor block) was collected from each case, and three 1-mm-diameter tumor cores were obtained with a trephine apparatus (Seoungkohn, Seoul, Korea). Trephinated paraffin tissue cores were consecutively placed in recipient plastic molds (tissue array mold; Seoungkohn), which were filled with liquid paraffin and cooled.

For the automatic immunohistochemistry staining device (Benchmark XT, Ventana Medical System, Tucson, AZ, USA), a protocol using formalin-fixed, paraffin-embedded tissue sections was used. Briefly, 5 - $\mu$ m-thick sections were transferred onto poly-Llysine-coated adhesive slides and dried at $62{ }^{\circ} \mathrm{C}$ for $30 \mathrm{~min}$. After standard heat epitope retrieval for 30 min in ethylene diamine tetra-acetic acid ( $\mathrm{pH}$ 8.0) in the autostainer, the samples were incubated with antibodies against cleaved MET (dilution 1:50) and MST1R (dilution 1:25; both from Santa Cruz Biotechnology, Santa Cruz, CA, USA). The sections were subsequently incubated with biotinylated antirabbit immunoglobulins, peroxidase-labeled streptavidin (LSAB kit, DAKO, Glostrup, Denmark), and 3,3'-diaminobenzidine. Slides were counterstained with Harris hematoxylin.

The expression of MET or MST1R was evaluated using the criteria suggested by Xu et al. ${ }^{32}$ A sample was considered MET or MST1R positive if $>30 \%$ of definitive HRS cells showed immunohistochemical reactivity with MET and MST1R antibodies (Figures 1a and b, respectively). A sample was considered MET or MST1R negative if MET or MST1R expression was negative or $<30 \%$ positive for HRS cells, or positive for bystander cells only. The median number of tumor cells for each case was 24. All interpretations of MET and MST1R staining patterns were performed by a colleague blinded to knowledge of the clinical outcomes.

The in situ hybridization (ISH) analysis for EBV-encoded RNA-1 and RNA-2 (EBER) was performed and scored as described elsewhere. ${ }^{35}$

\section{mRNA ISH}

RNA ISH was conducted using a commercially available mRNA ISH kit (QuantiGeneViewRNA, Paranomics, Fremont, CA, USA) according to the manufacturer's instructions. To remove the paraffin from the samples, the slides were deparaffinized in xylene. The samples were incubated with a pretreatment solution followed by protease digestion. MET or MST1R gene-specific probe was designed. A probe set was hybridized and amplifier molecules were hybridized to each pair of oligonucleotides. The fast red substrate (MET prove) and the fast blue substrate (MST1R prove), alkaline phophatase breaks down the substrate to form a precipitate. The slides were counterstained with hematoxylin.

The expression level was semiquantitatively evaluated based on the number of blue or red dots in the 

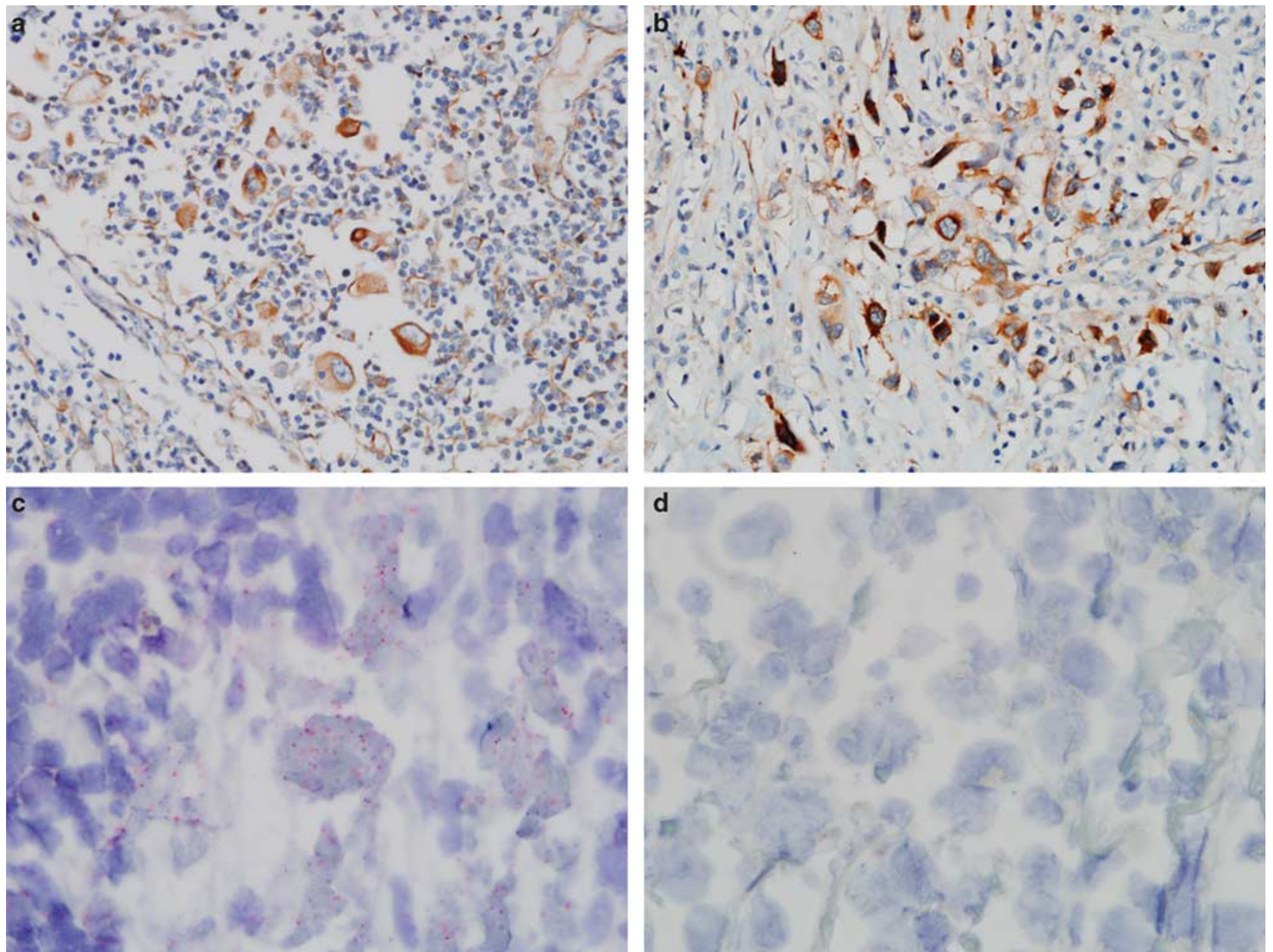

Figure 1 MET and MST1R expression in cHL tissue. (a) HRS cells showing strong MET immunostaining on the cell membranes (original magnification $\times 400$ ). (b) MST1R expression on the cell membranes (original magnification $\times 400$ ). (c) High expression of MET mRNA (red dot) and MST1R mRNA (blue dot) on tumor cells (original magnification $\times 1,000$ ). (d) No MET/MST1R mRNA expression on tumor cells (original magnification $\times 1,000$ ).

tumor cells $(0,1+, 2+$, and $3+)$. In normal lymphoid tissue, the expression of MET/MST1R mRNA was $1+$. For the purpose of further analysis, the data were grouped into two categories using a cutoff value equal to the MET/MST1R mRNA level in normal lymphoid tissue: negativity $(0$ and $1+)$ and positivity $(2+$ and $3+$ ) (Figures $1 \mathrm{c}$ and $\mathrm{d}$ ).

\section{Statistical Analysis}

Overall survival (OS) was defined as the time between the first day of diagnosis and the date of death from any cause. The follow-up of patients still alive was censored at their last follow-up date. Event-free survival (EFS) was defined as the interval between the first day of diagnosis and the date of disease progression, relapse, or death from any cause. The follow-up of patients still alive without event was censored at their last follow-up date. The OS and EFS were analyzed with Kaplan-Meier curves, which were compared by log-rank testing.
Multivariate prognostic analyses were performed on OS and EFS with the Cox proportional hazards regression model. Categorical variables were compared using the $\chi^{2}$ test. All statistical analyses were performed by the SPSS statistical software program (version 18.0; SPSS, Chicago, IL, USA). $P<0.05$ was considered statistically significant.

\section{Results}

\section{Patient Characteristics}

The clinical characteristics of the 100 patients included in the present study are summarized in Table 1. Patient age ranged from 4 to 77 years (median, 32 years). We recorded 36 patients experiencing relapse, disease progression, or death. Median OS and EFS were not reached. The estimated 5-year OS and EFS were 81.1 and $61.9 \%$, respectively. 
Table 1 Demographic and clinical characteristics of patients

\begin{tabular}{lc}
\hline Characteristic at diagnosis & No. of patients (\%) \\
\hline Age, median (range, years) & $33(4-77)$ \\
Male gender & $57(57 \%)$ \\
Histologic subtype & \\
Nodular sclerosis & $61(61 \%)$ \\
Mixed cellularity & $24(24 \%)$ \\
Lymphocyte rich & $7(7 \%)$ \\
Lymphocyte depleted & $2(2 \%)$ \\
Not classifiable & $6(6 \%)$ \\
Ann Arbor stage & \\
I & $15(15 \%)$ \\
II & $30(30 \%)$ \\
III & $30(30 \%)$ \\
IV & $25(25 \%)$ \\
Stage (limited vs advanced) & \\
Limited & $41(41 \%)$ \\
Advanced & $59(59 \%)$ \\
B symptoms present & $38(38 \%)$ \\
International Prognostic Score $\geq 4$ (high risk) & $16(16 \%)$ \\
EBER positivity & $48(48 \%)$ \\
Primary treatment & \\
Chemotherapy & \\
Chemoradiotherapy & $64(63.4 \%)$ \\
Radiotherapy only & $34(33.7 \%)$ \\
Chemotherapeutic regimen & $3(3 \%)$ \\
ABVD & \\
ABVD/C-MOPP hybrid & \\
BEACOPP & $68(68 \%)$ \\
C-MOPP & $70(10 \%)$ \\
Other regimen & $6(6 \%)$ \\
\hline
\end{tabular}

EBER, Epstein-Barr virus-encoded RNA-1 and RNA-2 assessed by in situ hybridization; ABVD, doxorubicin, bleomycin, vinblastine, and dacarbazine; BEACOPP, bleomycin, etoposide, doxorubicin, cyclophosphamide, vincristine, procarbazine, and prednisone; C-MOPP, cyclophosphamide, vincristine, prednisone, and procarbazine.

\section{MET Protein Expression in cHL Tissue}

Thirty-eight patients (38\%) showed cytoplasmic and membranous positivity for MET. The clinicopathological factors of patients according to MET expression are summarized in Table 2. The MET-negative patients were more likely to have the NS subtype ( 67.7 vs $50 \%$ ) and less likely to have the LR subtype (3.2 vs $13.2 \% ; P=0.026$ ) than MET-positive patients. No distinction between the groups was observed in terms of age $(P=0.211)$, sex $(P=0.303)$, B symptoms $(P=0.396)$, Ann Arbor stage $(P>0.999)$, IPS $(P=0.09)$, LDH $(P=0.666)$, EBER $(P=0.412)$, bulky disease $(P=0.498)$, treatment method $(P=0.631)$, or primary chemotherapeutic regimen $(P=0.354)$.

\section{MST1R Protein Expression in cHL Tissue}

Twenty-six patients (26\%) showed cytoplasmic and membranous positivity for MST1R. The clinicopathological factors of patients according to MST1R expression are summarized in Table 2. MST1Rpositive patients were more likely to have the NS subtype (80.8 vs $54.1 \%$ ), and were less likely to have the LR subtype (0 vs 9.5\%; $P=0.128$ ) than MST1Rnegative patients. No distinction between the groups was observed in terms of age $(P=0.353)$, sex $(P=0.819)$, B symptoms $(P=0.483)$, Ann Arbor stage $(P=0.644)$, IPS $(P=0.227)$, LDH $(P=0.625)$, EBER $(P=0.504)$, bulky disease $(P=0.121)$, treatment method $(P=0.401)$, or primary chemotherapeutic regimen $(P=0.582)$. Coexpression of MET and MST1R in tumor cells was observed in 13 cases (13\%). In all, 25 cases (25\%) showed MET expression only, 13 cases (13\%) showed MST1R expression only, and 49 cases were negative for both MET and MST1R.

\section{Correlation Between MET or MST1R Protein Expression and MET or MST1R mRNA Expression}

MET or MST1R protein expression was associated with high MET or MST1R mRNA expression, respectively (Table $3, P<0.001$ and $P=0.029$, respectively).

\section{Prognostic Significance of MET and MST1R Protein Expression}

MET-negative patients had lower 5-year OS rates than MET-positive patients ( 73.8 vs $93.7 \%, P=0.004$; Figure 2a), although the 5-year EFS rates were comparable (59.4 vs 65.4\%, $P=0.131$; Figure 2b). MST1R-negative patients had lower 5-year OS rates (76.1 vs 96.2\%, $P=0.022$; Figure 3a) and 5-year EFS rates (55.6 vs $81.4 \%, P=0.021$; Figure $3 \mathrm{~b}$ ) than MST1R-positive patients. To evaluate the relative importance of MET and MST1R, we combined the dichotomized MET and MST1R, and stratified patients into four groups (MET +/MST1R +, MET + /MST1R - , MET - /MST1R +, and MET - / MST1R -). Patients with MET +/MST1R + had better OS $(P=0.004$; Figure 4a) and EFS rates $(P=0.085$; Figure 4b) compared with the other expression patterns, although the statistical significance of the EFS was marginal.

To further assess the additional prognostic information regarding MET and MST1R, we performed subgroup analyses according to the Ann Arbor stage. In advanced-stage disease, MET-negative cases showed worse OS rates than MET-positive cases $(P=0.002$; Supplementary Figure 1b). However, in limited-stage disease, MET expression was not significantly associated with OS ( $P=0.619$; Supplementary Figure 1a). In both stages, MET expression was not significantly associated with EFS $(P=0.452$ for limited stage; Supplementary Figure 1c and $P=0.212$ for advanced stage; Supplementary Figure 1d).

In patients with advanced-stage disease, MST1Rnegative cases showed a worse trend for OS rate compared with MST1R-positive cases $(P=0.071$; Supplementary Figure 2b), but MST1R expression was not significantly associated with OS rate in patients with limited-stage disease $(P=0.178$; Supplementary Figure 2a). For advanced-stage 
Table 2 Correlation of MET and MST1R protein expression with clinicopathologic variables

\begin{tabular}{|c|c|c|c|c|c|c|}
\hline \multirow{2}{*}{ Characteristic } & \multicolumn{2}{|c|}{ MET expression } & \multirow{2}{*}{ P-value } & \multicolumn{2}{|c|}{ MST1R expression } & \multirow{2}{*}{ P-value } \\
\hline & Negative $(\mathrm{n}=62)$ & Positive $(\mathrm{n}=38)$ & & Negative $(\mathrm{n}=74)$ & Positive $(\mathrm{n}=26)$ & \\
\hline Age & & & $0.211^{\mathrm{a}}$ & & & $0.353^{\mathrm{a}}$ \\
\hline Age $<40$ & $34(54.8 \%)$ & $26(68.4 \%)$ & & $42(56.8 \%)$ & $18(69.2 \%)$ & \\
\hline Age $\geq 40$ & $28(45.2 \%)$ & $12(31.6 \%)$ & & $32(43.2 \%)$ & $8(20.8 \%)$ & \\
\hline Gender & & & $0.303^{\mathrm{a}}$ & & & $0.819^{\mathrm{a}}$ \\
\hline Male & $38(61.3 \%)$ & $19(50 \%)$ & & $43(58.1 \%)$ & $14(53.8 \%)$ & \\
\hline Female & $24(38.7 \%)$ & $19(50 \%)$ & & $31(41.9 \%)$ & $12(46.2 \%)$ & \\
\hline Disease subtype & & & $0.026^{\mathrm{b}}$ & & & $0.128^{\mathrm{b}}$ \\
\hline Nodular sclerosis & $42(67.7 \%)$ & $19(50 \%)$ & & $40(54.1 \%)$ & $21(80.8 \%)$ & \\
\hline Mixed cellularity & $16(25.8 \%)$ & $8(21.1 \%)$ & & $19(25.7 \%)$ & $5(19.2 \%)$ & \\
\hline Lymphocyte rich & $2(3.2 \%)$ & $5(13.2 \%)$ & & $7(9.5 \%)$ & $0(0 \%)$ & \\
\hline Lymphocyte depleted & $1(1.6 \%)$ & $1(2.6 \%)$ & & $2(2.7 \%)$ & $0(0 \%)$ & \\
\hline Not classifiable & $1(1.6 \%)$ & $5(13.2 \%)$ & & $6(8.1 \%)$ & $0(0 \%)$ & \\
\hline B symptom & & & $0.396^{\mathrm{a}}$ & & & $0.483^{\mathrm{a}}$ \\
\hline Absent & $36(58.1 \%)$ & $26(68.4 \%)$ & & $44(59.5 \%)$ & $18(69.2 \%)$ & \\
\hline Present & $26(41.9 \%)$ & $12(31.6 \%)$ & & $30(40.5 \%)$ & $8(30.8 \%)$ & \\
\hline Ann Arbor stage & & & $>0.999^{\mathrm{a}}$ & & & $0.644^{\mathrm{a}}$ \\
\hline Limited & $25(40.3 \%)$ & $16(42.1 \%)$ & & $29(39.2 \%)$ & $12(46.2 \%)$ & \\
\hline Advanced & $37(59.7 \%)$ & $22(57.9 \%)$ & & $45(60.8 \%)$ & $14(53.8 \%)$ & \\
\hline IPS & & & $0.09^{\mathrm{a}}$ & & & $0.227^{\mathrm{b}}$ \\
\hline$<4$ & $49(79 \%)$ & $35(92.1 \%)$ & & $60(81.1 \%)$ & $24(92.3 \%)$ & \\
\hline$\geq 4$ & $13(21 \%)$ & $3(7.9 \%)$ & & $14(18.9 \%)$ & $2(7.7 \%)$ & \\
\hline$L D H(U / I)$ & & & $0.666^{\mathrm{a}}$ & & & $0.625^{\mathrm{a}}$ \\
\hline$<250$ & $19(32.2 \%)$ & $14(36.8 \%)$ & & $23(31.9 \%)$ & $10(40 \%)$ & \\
\hline$\geq 250$ & $40(67.8 \%)$ & $24(63.2 \%)$ & & $49(68.1 \%)$ & $15(60 \%)$ & \\
\hline EBER & & & $0.412^{\mathrm{a}}$ & & & $0.504^{\mathrm{a}}$ \\
\hline Negative & $30(48.4 \%)$ & $22(57.9 \%)$ & & $40(54.1 \%)$ & $12(46.2 \%)$ & \\
\hline Positive & $32(51.6 \%)$ & $16(42.1 \%)$ & & $34(45.9 \%)$ & $14(53.8 \%)$ & \\
\hline Bulky disease & & & $0.498^{\mathrm{b}}$ & & & $0.121^{\mathrm{b}}$ \\
\hline Absent & $57(91.9 \%)$ & $33(86.8 \%)$ & & $69(93.2 \%)$ & $21(80.8 \%)$ & \\
\hline Present & $5(8.1 \%)$ & $5(13.2 \%)$ & & $5(6.8 \%)$ & $5(19.2 \%)$ & \\
\hline Primary treatment & & & $0.631^{\mathrm{b}}$ & & & $0.401^{\mathrm{b}}$ \\
\hline Chemotherapy & $39(62.9 \%)$ & $24(63.2 \%)$ & & $49(66.2 \%)$ & $14(53.8 \%)$ & \\
\hline Chemoradiotherapy & $22(35.5 \%)$ & $12(31.6 \%)$ & & $23(31.1 \%)$ & $11(42.3 \%)$ & \\
\hline Radiotherapy & $1(1.6 \%)$ & $2(5.3 \%)$ & & $2(2.7 \%)$ & $1(3.8 \%)$ & \\
\hline Primary chemotherapeutic regimen & & & $0.354^{\mathrm{b}}$ & & & $0.582^{b}$ \\
\hline ABVD & $38(62.3 \%)$ & $30(83.3 \%)$ & & $48(66.7 \%)$ & $20(80 \%)$ & \\
\hline ABVD/C-MOPP hybrid & $8(13.1 \%)$ & $2(5.6 \%)$ & & $8(11.1 \%)$ & $2(8 \%)$ & \\
\hline BEACOPP & $5(8.2 \%)$ & $2(5.6 \%)$ & & $6(8.3 \%)$ & $1(4 \%)$ & \\
\hline C-MOPP & $5(8.2 \%)$ & $1(2.8 \%)$ & & $6(8.3 \%)$ & $0(0 \%)$ & \\
\hline Other regimen & $5(8.2 \%)$ & $1(2.8 \%)$ & & $4(5.6 \%)$ & $2(8 \%)$ & \\
\hline
\end{tabular}

IPS, International Prognostic Score; LDH, lactate dehydrogenase; EBER, Epstein-Barr virus-encoded RNA-1 and RNA-2 assessed by in situ hybridization; ABVD, doxorubicin, bleomycin, vinblastine, and dacarbazine; BEACOPP, bleomycin, etoposide, doxorubicin, cyclophosphamide, vincristine, procarbazine, and prednisone; C-MOPP, cyclophosphamide, vincristine, prednisone, and procarbazine.

${ }^{\mathrm{a}}$ The $\chi^{2}$ test by two-sided Pearson's test.

${ }^{\mathrm{b}}$ The $\chi^{2}$ test by two-sided Fisher's test.

Table 3 Correlation of MET and MST1R protein expression with mRNA expression

\begin{tabular}{|c|c|c|c|c|c|c|c|}
\hline \multirow[t]{2}{*}{ Characteristic } & \multicolumn{2}{|c|}{ MET expression } & \multirow[t]{2}{*}{$\mathrm{P}$-value } & & \multicolumn{2}{|c|}{ MST1R expression } & \multirow[t]{2}{*}{ P-value } \\
\hline & Negative $(\mathrm{n}=62)$ & Positive $(\mathrm{n}=38)$ & & & Negative $(\mathrm{n}=74)$ & Positive $(\mathrm{n}=26)$ & \\
\hline MET mRNA & & & $<0.001^{\mathrm{a}}$ & MST1R mRNA & & & $0.029^{\mathrm{a}}$ \\
\hline Negative & $56(90.3 \%)$ & $23(60.5 \%)$ & & Negative & $55(74.3 \%)$ & $13(53.6 \%)$ & \\
\hline Positive & $6(9.7 \%)$ & $15(39.5 \%)$ & & Positive & $19(25.7 \%)$ & $13(46.4 \%)$ & \\
\hline
\end{tabular}

aThe $\chi^{2}$ test by two-sided Pearson's test. 

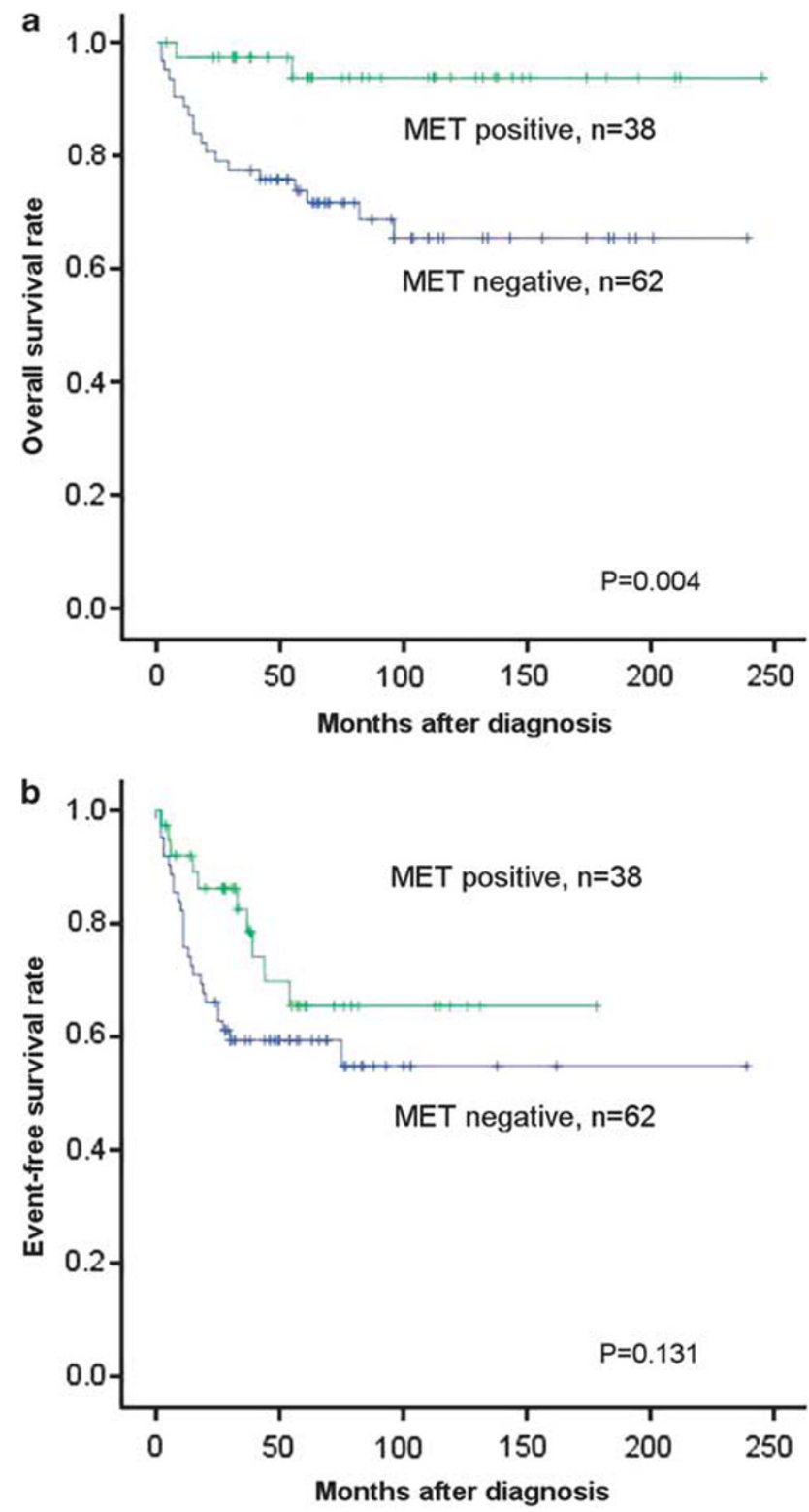

Figure 2 Comparison of survival rates according to MET and MST1R protein expression. (a) Overall survival according to MET. (b) Event-free survival according to MET.

disease, MST1R-negative cases showed worse EFS rates than MST1R-positive cases $(P=0.041$; Supplementary Figure 2d). For limited-stage disease, MST1R expression was not significantly associated with EFS rate $(P=0.336$; Supplementary Figure 2c).

In univariate analysis, both OS and EFS were associated with IPS $(\geq 4)$. Age ( $>40$ years), B symptoms, advanced stage, abnormal LDH, and ABVD-based chemotherapeutic regimen (doxorubicin, bleomycin, vinblastine, and dacarbazine) were associated with OS but not with EFS (Table 4). In multivariate analysis, MET expression was an independent prognostic marker for OS, along with high-risk IPS $(\geq 4)$, B symptoms, age ( $>40$ years),
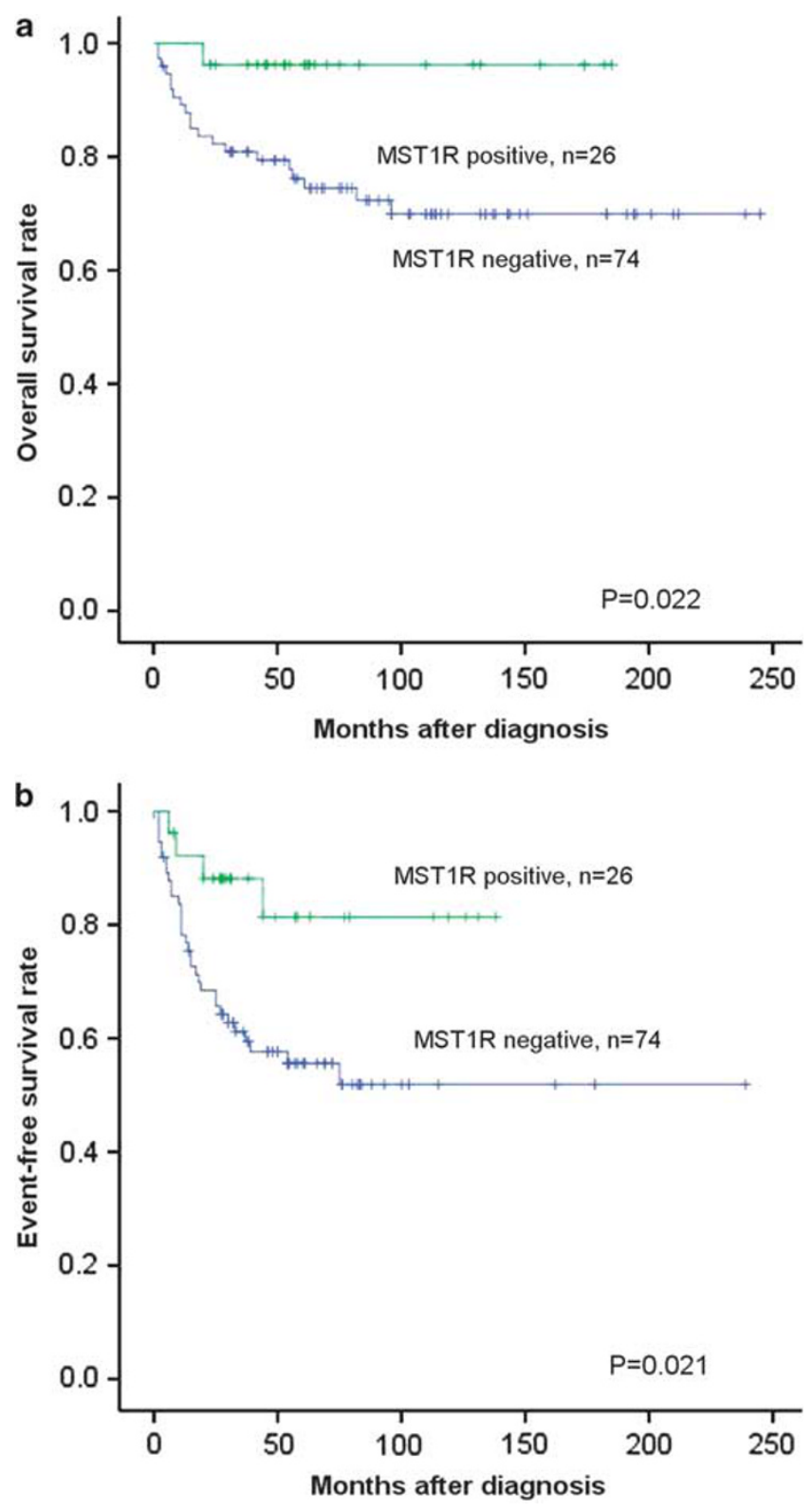

Figure 3 Comparison of survival rates according to MST1R protein expression. (a) Overall survival. (b) Event-free survival.

abnormal LDH, and ABVD-based chemotherapeutic regimen (Table 5). In multivariate analysis, MST1R expression was an independent prognostic marker for EFS, along with high-risk IPS $(\geq 4)$, B symptoms, age ( $>40$ years), abnormal $\mathrm{LDH}$, and ABVD-based chemotherapeutic regimen (Table 6).

\section{Prognostic Significance of MET and MST1R mRNA Expression}

Although there was no statistically significant difference, patients with MET positivity had a trend toward better OS than the MET-negative group (5-year OS rate: 89.9 vs $78.6 \%, \quad P=0.361$; Supplementary 

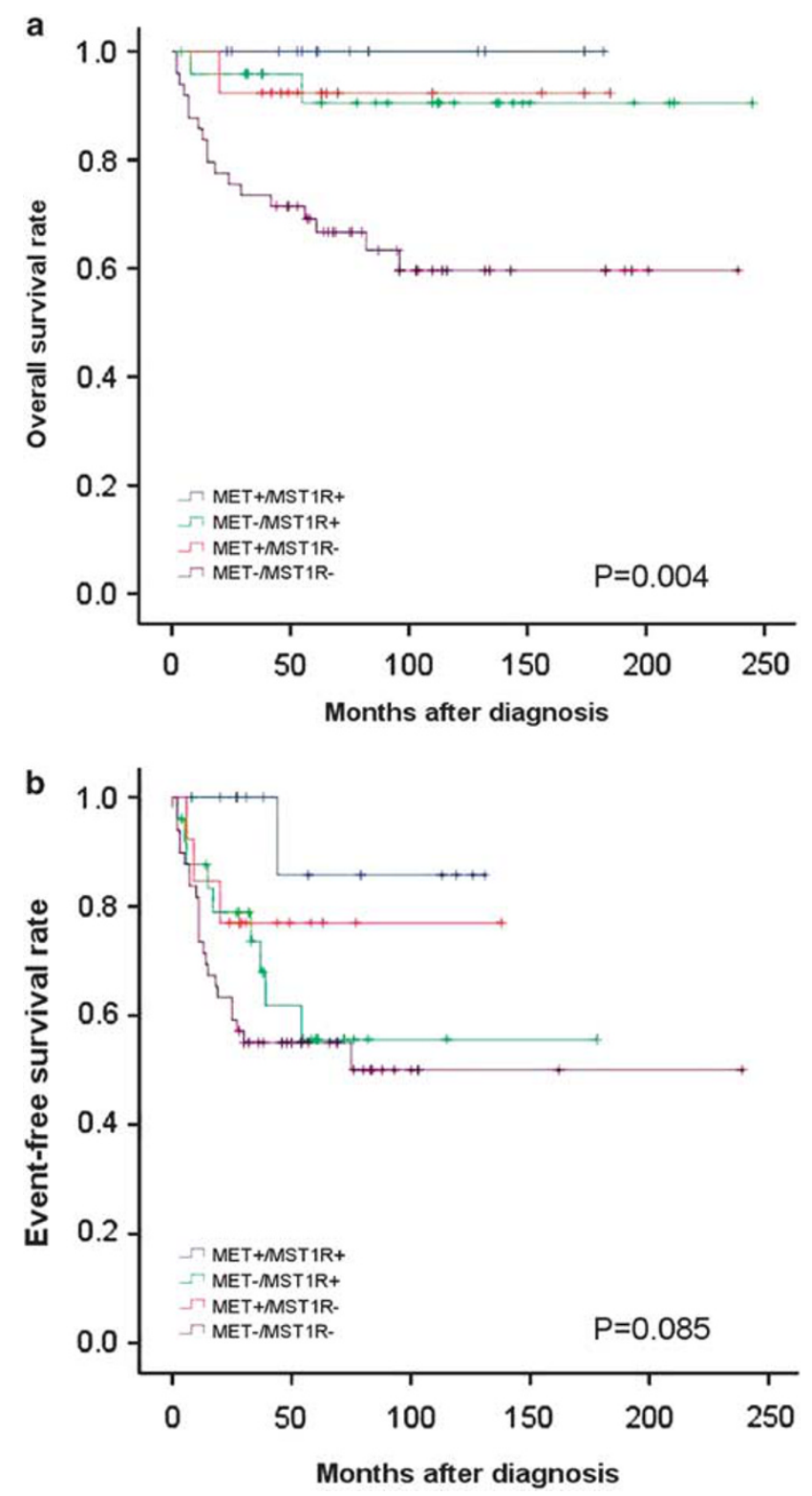

Figure 4 Comparison of survival rates according to MET/MST1R protein expression pattern. (a) Overall survival. (b) Event-free survival.

Figure 3a). Patients with MST1R positivity also had a trend toward better OS than the MST1R-negative group (5-year OS rate: 86.9 vs $78.3 \%, P=0.367$; Supplementary Figure 3c), although it was statistically nonsignificant. The MET or MST1R mRNA expression was not significantly associated with EFS $(P=0.772$; Supplementary Figure $3 \mathrm{~b}$ and $P=0.897$; Supplementary Figure 3d, respectively).

\section{Discussion}

We found that the expression of MET, MST1R, or both was significantly associated with favorable clinical outcomes, which supports the hypothesis that a MET/MST1R-related signaling pathway plays an important role in the progression of cHL. Evaluation of the MST1R and MET expression status may aid in selecting patients who could benefit from intensive therapy. Limitations of this study include the retrospective nature of the study design, the short follow-up period, and the relatively small sample size.

In striking contrast to our results, expression of MET/HGF in solid malignancies has been associated with unfavorable clinical outcomes, $16,17,19,20,22,25$ although a few studies identified a favorable prognostic impact of MET. ${ }^{36,37}$ Expression of MST1R is also associated with unfavorable clinical outcomes $^{38-40}$ or not associated with prognosis. ${ }^{41}$ However, a recent study found that MET expression is associated with a favorable prognosis in cHL. ${ }^{32}$ These findings in cHL suggest that the prognostic impact of MET and MST1R in CHL may be significantly different from those in other solid malignancies.

There are several possible explanations for the favorable outcome associated with MET expression in patients with cHL. The unique histological features of cHL could play an important role. Because cHL is composed, on average, of $<1 \%$ malignant HRS cells and a majority of reactive cells, including $\mathrm{T}$ and $\mathrm{B}$ lymphocytes and other cell types, the reactive cells could play an important role in disease progression. $\mathrm{Xu}$ et $\mathrm{al}^{32}$ suggested that the activation of MET in HRS cells might change the immunosuppressive function of the reactive cells by causing the secretion of factors that alter the microenvironment. This process could result in a decreased risk of cHL progression. In a mouse model, HGF gene transfer attenuates acute liver injury and hepatic fibrosis ${ }^{42,43}$ and suppresses TGF- $\beta$ levels. ${ }^{44}$ HRS cells may produce TGF- $\beta$ to downmodulate the immune response. ${ }^{45}$ Therefore, MET expression in HRS cells could reduce TGF- $\beta$ production and help recover immune function. A previous study has revealed that MET inhibitor SU11274 suppressed cell growth by inducing G2/M cell cycle arrest in cHL cell line. ${ }^{32}$ Other studies have also found that MET inhibitor PHA665752 causes cell death and induces apoptosis in diffuse large B-cell lymphoma cell line. ${ }^{37}$ MST1R expression is also required for the growth of lymphoblastoid cell line and posttransplantation lymphoproliferative disorder. ${ }^{46}$

In a study of diffuse large B-cell lymphoma by Uddin et $a l,{ }^{37}$ the oncogenic roles of the MET and/or MST1R in promoting tumor cell growth and proliferation were reflected in the higher proliferation rates of the tumors that expressed MET protein. The authors ascribed the superior survival of diffuse large B-cell lymphoma cases with MET expression to the increased sensitivity to chemotherapy associated with higher proliferation rates (Uddin et $a{ }^{37}$ ). Although we did not examine the proliferation rates of HRS cells in this series, 
Table 4 Univariate analyses with MET or MST1R protein expression for overall survival (OS) and event-free survival (EFS)

Covariate

Univariate analysis

\begin{tabular}{|c|c|c|c|c|c|c|c|}
\hline & & \multicolumn{3}{|c|}{ OS } & \multicolumn{3}{|c|}{$E F S$} \\
\hline & & $H R$ & $95 \% C I$ & $\mathrm{P}$-value $\mathrm{a}^{\mathrm{a}}$ & $H R$ & $95 \% C I$ & $\mathrm{P}$-value $\mathrm{a}^{\mathrm{a}}$ \\
\hline Age (years) & $<40$ vs $\geq 40$ & 3.823 & $1.48-9.85$ & 0.006 & 1.549 & $0.80-2.97$ & 0.19 \\
\hline Sex & Female vs male & 2.717 & $0.99-7.43$ & 0.052 & 1.172 & $0.60-2.27$ & 0.639 \\
\hline B symptoms & $(-) v s(+)$ & 2.86 & $1.18-6.9$ & 0.02 & 1.429 & $0.74-2.75$ & 0.288 \\
\hline IPS & $<4$ vs $\geq 4$ & 4.719 & $1.98-11.2$ & $<0.001$ & 2.137 & $1.00-4.55$ & 0.049 \\
\hline Ann Arbor stage & Limited vs advanced & 3.165 & $1.06-9.4$ & 0.038 & 1.801 & $0.88-3.66$ & 0.104 \\
\hline Bulky disease & $<10$ vs $\geq 10$ & 2.151 & $0.28-16.0$ & 0.455 & 1.152 & $0.35-3.75$ & 0.815 \\
\hline $\mathrm{LDH}(\mathrm{U} / \mathrm{l})$ & Normal vs abnormal & 5.168 & $1.19-22.2$ & 0.028 & 1.616 & $0.75-3.45$ & 0.216 \\
\hline EBER & $(-) v s(+)$ & 1.277 & $0.63-2.56$ & 0.491 & 0.948 & $0.58-1.53$ & 0.827 \\
\hline MET expression & $(-)$ vs $(+)$ & 0.156 & $0.03-0.67$ & 0.012 & 0.576 & $0.27-1.19$ & 0.138 \\
\hline MST1R expression & $(-)$ vs $(+)$ & 0.136 & $0.01-1.01$ & 0.052 & 0.316 & $0.11-0.08$ & 0.03 \\
\hline Chemo. regimen & Non-ABVD vs ABVD & 0.415 & $0.17-0.97$ & 0.044 & 0.895 & $0.44-1.81$ & 0.758 \\
\hline
\end{tabular}

HR, hazard ratio; CI, confidence interval; IPS, International Prognostic Score; LDH, lactate dehydrogenase; EBER, Epstein-Barr virus-encoded RNA-1 and RNA-2 assessed by in situ hybridization; Chemo, chemotherapeutic; ABVD, doxorubicin, bleomycin, vinblastine, and dacarbazine.

${ }^{\mathrm{a}}$ Cox univariate analysis.

Table 5 Multivariate analysis with MET protein expression for overall survival (OS) and event-free survival (EFS)

\begin{tabular}{|c|c|c|c|c|c|c|}
\hline \multirow{2}{*}{ Prognostic factor } & \multicolumn{3}{|c|}{$O S$} & \multicolumn{3}{|c|}{ EFS } \\
\hline & $\mathrm{P}$-value & $H R$ & $95 \% C I$ & $\mathrm{P}$-value & $H R$ & $95 \% C I$ \\
\hline MET positive & 0.025 & 0.187 & $0.043-0.811$ & 0.203 & 0.62 & $0.297-1.295$ \\
\hline IPS $\geq 4$ & 0.003 & 3.815 & $1.597-9.113$ & 0.087 & 1.949 & $0.908-4.185$ \\
\hline MET positive & 0.015 & 0.163 & $0.038-0.702$ & 0.151 & 0.585 & $0.282-1.215$ \\
\hline Presence of B symptoms & 0.03 & 2.654 & $1.098-6.419$ & 0.356 & 1.364 & $0.706-2.637$ \\
\hline MET positive & 0.02 & 0.176 & $0.041-0.76$ & 0.171 & 0.599 & $0.288-1.247$ \\
\hline Age $\geq 40$ & 0.01 & 3.484 & $1.348-9.002$ & 0.201 & 1.534 & $0.796-2.957$ \\
\hline MET positive & 0.015 & 0.163 & $0.038-0.701$ & 0.127 & 0.564 & $0.271-1.176$ \\
\hline LDH abnormal & 0.032 & 4.947 & $1.146-21.34$ & 0.214 & 1.619 & $0.757-3.462$ \\
\hline MET positive & 0.022 & 0.179 & $0.041-0.78$ & 0.152 & 0.579 & $0.274-1.223$ \\
\hline ABVD regimen & 0.166 & 0.542 & $0.228-1.289$ & 0.95 & 1.023 & $0.494-2.119$ \\
\hline
\end{tabular}

IPS, International Prognostic Score; LDH, lactate dehydrogenase; EBV, Epstein-Barr virus; HR, hazard ratio; CI, confidence interval; ABVD, doxorubicin, bleomycin, vinblastine, and dacarbazine.

Table 6 Multivariate analysis with MST1R protein expression for overall survival (OS) and event-free survival (EFS)

\begin{tabular}{|c|c|c|c|c|c|c|}
\hline \multirow{2}{*}{ Prognostic factor } & \multicolumn{3}{|c|}{ OS } & \multicolumn{3}{|c|}{ EFS } \\
\hline & $\mathrm{P}$-value & $H R$ & $95 \% C I$ & $\mathrm{P}$-value & $H R$ & $95 \% C I$ \\
\hline MST1R positive & 0.074 & 0.159 & $0.021-1.193$ & 0.04 & 0.335 & $0.118-0.951$ \\
\hline IPS $\geq 4$ & 0.001 & 4.149 & $1.743-9.876$ & 0.095 & 1.908 & $0.893-4.078$ \\
\hline MST1R positive & 0.058 & 0.144 & $0.019-1.07$ & 0.032 & 0.321 & $0.113-0.909$ \\
\hline Presence of B symptoms & 0.028 & 2.687 & $1.113-6.492$ & 0.379 & 1.344 & $0.696-2.597$ \\
\hline MST1R positive & 0.07 & 0.156 & $0.021-1.163$ & 0.035 & 0.326 & $0.115-0.923$ \\
\hline Age $\geq 40$ & 0.008 & 3.593 & $1.392-9.272$ & 0.209 & 1.522 & $0.791-2.93$ \\
\hline MST1R positive & 0.073 & 0.158 & $0.021-1.185$ & 0.047 & 0.347 & $0.122-0.986$ \\
\hline LDH abnormal & 0.034 & 4.852 & $1.125-20.92$ & 0.307 & 1.487 & $0.694-3.186$ \\
\hline MST1R positive & 0.071 & 0.157 & $0.021-1.174$ & 0.035 & 0.323 & $0.113-0.922$ \\
\hline ABVD regimen & 0.088 & 0.473 & $0.200-1.117$ & 0.933 & 1.031 & $0.505-2.108$ \\
\hline
\end{tabular}

IPS, International Prognostic Score; LDH, lactate dehydrogenase; EBV, Epstein-Barr virus; HR, hazard ratio; CI, confidence interval; ABVD, doxorubicin, bleomycin, vinblastine, and dacarbazine.

previous studies have reported prognostic relevance of proliferation rates of HRS cells in $\mathrm{CHL}{ }^{47-49}$ It is conceivable that the superior survival of cHL with
MET and/or MST1R expression in the present study may also involve higher proliferation rate of HRS cells. 
In our study, MET or MST1R protein correlated with each level of mRNA. However, MET or MST1R mRNA expression was not associated with the survival outcome of patients. Alternative splicing was reported in MET and MST1R, and this process could result in the different expression of protein. ${ }^{50}$ MET protein levels are affected by various factors including the levels of MET mRNA expression and stability; the translational activity, which may be regulated by exogenous and endogenous microRNAs; and proteasomal degradation. ${ }^{51-53}$ Therefore, several protein regulating factors may be responsible for the differences between protein and mRNA levels.

Amplification, mutation, and overexpression of MET were described in various malignancies and such dysregulation was also associated with tumor progression. ${ }^{54,55}$ Amplification, mutation, and overexpression of the MST1R have also been reported in renal and lung cancer and implicated in transformation, tumor formation, and metastasis. ${ }^{39,56}$ Furthermore, amplification of $3 p$, which is a the chromosome region containing MST1R, is a common event in lung, renal, and breast cancer, occurring in $15-42.5 \%$ of the samples examined. ${ }^{57}$ MET signaling is mainly mediated by the RASMAPK and PI3K-AKT pathways and affects gene expression and cell cycle progression. ${ }^{58,59} \mathrm{Xu}$ et $a l^{32}$ also reported that HGF stimulation induced p-AKT upregulation in cHL cell line. MST1R is also a strong inducer of both MAPK and the PI3K signaling pathways. ${ }^{60}$ Therefore, coexpression of MET and MST1R finally would result in tumor progression.

Intriguingly, patients with coexpression of MST1R and MET showed better survival outcomes than those with MET or MST1R expression alone, or no expression. MST1R and MET are expressed on the cell membrane surface as preformed dimers before ligand stimulation. A bidirectional transphosphorylation occurs between MET and MST1R after exposure of cells to either HGF or macrophagestimulating protein. ${ }^{61}$ Although MST1R is less efficient than MET as a kinase, the formation of MET/MST1R complexes leads to a more efficient MST1R transphosphorylation by MET, resulting in a more sustained signal than that induced by the MST1R/MST1R homodimer. Activation of both MET and MST1R may induce a cooperative or synergistic response to their ligands. Patients with tumors coexpressing MET and MST1R reportedly have a worse prognosis than those with single receptor-positive tumors. ${ }^{34,62,63}$ The synergistic effect of MET and MST1R might induce a favorable outcome, as MET expression is associated with a better prognosis in cHL.

A previous study demonstrated a correlation of MET expression with EBV positivity in cHL patients, ${ }^{30}$ whereas a larger cohort showed no association. ${ }^{64}$ In the present study, we observed no correlation between MET expression and EBV status, in agreement with the data of previous studies. ${ }^{32,64}$ No studies have examined the correlation between
MST1R expression and EBV status in cHL patients. Our result found no correlation between MST1R expression and EBV status. These results suggest that MET and MST1R expression was associated with survival outcome independent of EBV status.

In the present study, MET or MST1R expression was significantly associated with better prognosis, especially for patients with advanced-stage disease, which is consistent with results from another study in patients with cHL. ${ }^{32}$ Although IPS is the standard stratification system for survival in patients with advanced-stage disease, it is limited to selecting distinct subgroups of patients at very high risk of recurrence. ${ }^{65}$ This result suggests that MET or MST1R positivity provides additional prognostic information, independent of IPS.

In summary, our results suggest the prognostic significance of the coexpression of MST1R and MET in CHL patients with advanced-stage disease. Coexpression of MST1R and MET can be used to identify a subgroup of cHL patients at high risk for recurrence or progression who may benefit from aggressive chemotherapy. Further studies, including prospective clinical trials, are needed to investigate the effects of MET or MST1R expression on clinical outcomes and to confirm the present findings.

\section{Acknowledgements}

This study was supported by a grant (2011-090) from the Asan Institute for Life Sciences, Seoul, South Korea.

\section{Disclosure/conflict of interest}

The authors declare no conflict of interest.

\section{References}

1 Joos S, Kupper M, Ohl S, et al. Genomic imbalances including amplification of the tyrosine kinase gene JAK2 in CD30 + Hodgkin cells. Cancer Res 2000;60:549-552.

2 Weniger MA, Melzner I, Menz CK, et al. Mutations of the tumor suppressor gene SOCS-1 in classical Hodgkin lymphoma are frequent and associated with nuclear phospho-STAT5 accumulation. Oncogene 2006;25:2679-2684.

3 Portis T, Dyck P, Longnecker R. Epstein-Barr Virus (EBV) LMP2A induces alterations in gene transcription similar to those observed in Reed-Sternberg cells of Hodgkin lymphoma. Blood 2003;102:4166-4178.

4 Vockerodt M, Morgan SL, Kuo M, et al. The EpsteinBarr virus oncoprotein, latent membrane protein-1, reprograms germinal centre B cells towards a Hodgkin's Reed-Sternberg-like phenotype. J Pathol 2008; 216:83-92.

5 Koh YW, Yoon DH, Suh C, et al. Impact of the EpsteinBarr virus positivity on Hodgkin's lymphoma in a large 
cohort from a single institute in Korea. Ann Hematol 2012;91:1403-1412.

6 Yoon DH, Koh YW, Kang HJ, et al. CD68 and CD163 as prognostic factors for Korean patients with Hodgkin lymphoma. Eur J Haematol 2012;88:292-305.

7 Koh YW, Kang HJ, Park C, et al. The ratio of the absolute lymphocyte count to the absolute monocyte count is associated with prognosis in Hodgkin's lymphoma: correlation with tumor-associated macrophages. Oncologist 2012;17:871-880.

8 Steidl C, Lee T, Shah SP, et al. Tumor-associated macrophages and survival in classic Hodgkin's lymphoma. N Engl J Med 2010;362:875-885.

9 Quddus F, Armitage JO. Salvage therapy for Hodgkin's lymphoma. Cancer J 2009;15:161-163.

10 Hasenclever D, Diehl V. A prognostic score for advanced Hodgkin's disease. International Prognostic Factors Project on Advanced Hodgkin's Disease. N Engl J Med 1998;339:1506-1514.

11 Gallamini A, Hutchings M, Rigacci L, et al. Early interim 2-[18F]fluoro-2-deoxy-D-glucose positron emission tomography is prognostically superior to international prognostic score in advanced-stage Hodgkin's lymphoma: a report from a joint ItalianDanish study. J Clin Oncol 2007;25:3746-3752.

12 Diepstra A, van Imhoff GW, Schaapveld M, et al. Latent Epstein-Barr virus infection of tumor cells in classical Hodgkin's lymphoma predicts adverse outcome in older adult patients. J Clin Oncol 2009;27: 3815-3821.

13 Ma PC, Maulik G, Christensen J, et al. c-Met: structure, functions and potential for therapeutic inhibition. Cancer Metastasis Rev 2003;22:309-325.

14 Benvenuti S, Comoglio PM. The MET receptor tyrosine kinase in invasion and metastasis. J Cell Physiol 2007;213:316-325.

15 Comoglio PM, Giordano S, Trusolino L. Drug development of MET inhibitors: targeting oncogene addiction and expedience. Nat Rev Drug Discov 2008;7:504-516.

16 Nakajima M, Sawada H, Yamada Y, et al. The prognostic significance of amplification and overexpression of C-met and C-erb B-2 in human gastric carcinomas. Cancer 1999;85:1894-1902.

17 Kammula US, Kuntz EJ, Francone TD, et al. Molecular co-expression of the c-Met oncogene and hepatocyte growth factor in primary colon cancer predicts tumor stage and clinical outcome. Cancer Lett 2007; 248:219-228.

18 Edakuni G, Sasatomi E, Satoh T, et al. Expression of the hepatocyte growth factor/c-Met pathway is increased at the cancer front in breast carcinoma. Pathol Int 2001;51:172-178.

19 Aune G, Lian AM, Tingulstad S, et al. Increased circulating hepatocyte growth factor (HGF): a marker of epithelial ovarian cancer and an indicator of poor prognosis. Gynecol Oncol 2011;121:402-406.

20 Tanimoto S, Fukumori T, El-Moula G, et al. Prognostic significance of serum hepatocyte growth factor in clear cell renal cell carcinoma: comparison with serum vascular endothelial growth factor. J Med Invest 2008;55:106-111.

21 Ma PC, Tretiakova MS, MacKinnon AC, et al. Expression and mutational analysis of MET in human solid cancers. Genes Chromosomes Cancer 2008;47: 1025-1037.

22 Ramirez R, Hsu D, Patel A, et al. Over-expression of hepatocyte growth factor/scatter factor (HGF/SF) and the $\mathrm{HGF} / \mathrm{SF}$ receptor (cMET) are associated with a high risk of metastasis and recurrence for children and young adults with papillary thyroid carcinoma. Clin Endocrinol (Oxf) 2000;53:635-644.

23 Ma PC, Jagadeeswaran R, Jagadeesh S, et al. Functional expression and mutations of c-Met and its therapeutic inhibition with SU11274 and small interfering RNA in non-small cell lung cancer. Cancer Res 2005;65: 1479-1488.

24 Kurzrock R, Sherman SI, Ball DW, et al. Activity of XL184 (Cabozantinib), an oral tyrosine kinase inhibitor, in patients with medullary thyroid cancer. J Clin Oncol 2011;29:2660-2666.

25 Masuya D, Huang C, Liu D, et al. The tumour-stromal interaction between intratumoral c-Met and stromal hepatocyte growth factor associated with tumour growth and prognosis in non-small-cell lung cancer patients. Br J Cancer 2004;90:1555-1562.

26 Chen YQ, Zhou YQ, Angeloni D, et al. Overexpression and activation of the RON receptor tyrosine kinase in a panel of human colorectal carcinoma cell lines. Exp Cell Res 2000;261:229-238.

27 Wang MH, Wang D, Chen YQ. Oncogenic and invasive potentials of human macrophage-stimulating protein receptor, the RON receptor tyrosine kinase. Carcinogenesis 2003;24:1291-1300.

28 Bardella C, Costa B, Maggiora P, et al. Truncated RON tyrosine kinase drives tumor cell progression and abrogates cell-cell adhesion through E-cadherin transcriptional repression. Cancer Res 2004;64: 5154-5161.

29 Wang MH, Kurtz AL, Chen Y. Identification of a novel splicing product of the RON receptor tyrosine kinase in human colorectal carcinoma cells. Carcinogenesis 2000;21:1507-1512.

30 Weimar IS, de Jong D, Muller EJ, et al. Hepatocyte growth factor/scatter factor promotes adhesion of lymphoma cells to extracellular matrix molecules via alpha 4 beta 1 and alpha 5 beta 1 integrins. Blood 1997;89:990-1000.

31 Renne C, Willenbrock K, Martin-Subero JI, et al. High expression of several tyrosine kinases and activation of the PI3K/AKT pathway in mediastinal large B cell lymphoma reveals further similarities to Hodgkin lymphoma. Leukemia 2007;21:780-787.

$32 \mathrm{Xu} \mathrm{C}$, Plattel W, van den Berg A, et al. Expression of the c-Met oncogene by tumor cells predicts a favorable outcome in classical Hodgkin's lymphoma. Haematologica 2012;97:572-578.

33 Chen Q, Seol DW, Carr B, et al. Co-expression and regulation of Met and Ron proto-oncogenes in human hepatocellular carcinoma tissues and cell lines. Hepatology 1997;26:59-66.

34 Cheng HL, Liu HS, Lin YJ, et al. Co-expression of RON and MET is a prognostic indicator for patients with transitional-cell carcinoma of the bladder. Br J Cancer 2005;92:1906-1914.

35 Huh J, Cho K, Heo DS, et al. Detection of Epstein-Barr virus in Korean peripheral T-cell lymphoma. Am J Hematol 1999;60:205-214.

36 Nakopoulou L, Gakiopoulou H, Keramopoulos A, et al. c-met tyrosine kinase receptor expression is associated with abnormal beta-catenin expression and favourable prognostic factors in invasive breast carcinoma. Histopathology 2000;36:313-325.

37 Uddin S, Hussain AR, Ahmed M, et al. Inhibition of c-MET is a potential therapeutic strategy for treatment 
of diffuse large B-cell lymphoma. Lab Invest 2010;90: 1346-1356.

38 Hui MK, Lai KK, Chan KW, et al. Prognostic significance of phosphorylated RON in esophageal squamous cell carcinoma. Med Oncol 2011;29:1699-1706.

39 Catenacci DV, Cervantes G, Yala S, et al. RON (MST1R) is a novel prognostic marker and therapeutic target for gastroesophageal adenocarcinoma. Cancer Biol Ther 2011;12:9-46.

40 Bishop EA, Lengyel ER, Yamada SD, et al. The expression of hepatocyte growth factor (HGF) and c-Met in uterine serous carcinoma. Gynecol Oncol 2011;121:218-223.

41 Zhou D, Pan G, Zheng C, et al. Expression of the RON receptor tyrosine kinase and its association with gastric carcinoma versus normal gastric tissues. BMC Cancer 2008;8:353.

42 Lin Y, Xie WF, Chen YX, et al. Treatment of experimental hepatic fibrosis by combinational delivery of urokinase-type plasminogen activator and hepatocyte growth factor genes. Liver Int 2005;25:796-807.

43 Xue F, Takahara T, Yata Y, et al. Attenuated acute liver injury in mice by naked hepatocyte growth factor gene transfer into skeletal muscle with electroporation. Gut 2002;50:558-562.

44 Ueki T, Kaneda Y, Tsutsui H, et al. Hepatocyte growth factor gene therapy of liver cirrhosis in rats. Nat Med 1999;5:226-230.

45 Poppema S, Potters M, Visser L, et al. Immune escape mechanisms in Hodgkin's disease. Ann Oncol 1998;9(Suppl 5):S21-S24.

46 Chou YC, Lin SJ, Lu J, et al. Requirement for LMP1induced RON receptor tyrosine kinase in Epstein-Barr virus-mediated B-cell proliferation. Blood 2011;118: 1340-1349.

47 Doussis-Anagnostopoulou IA, Vassilakopoulos TP, Thymara I, et al. Topoisomerase Ilalpha expression as an independent prognostic factor in Hodgkin's lymphoma. Clin Cancer Res 2008;14:1759-1766.

48 Dinand V, Malik A, Unni R, et al. Proliferative index and CD15 expression in pediatric classical Hodgkin lymphoma. Pediatr Blood Cancer 2008;50:280-283.

49 Tiemann M, Claviez A, Luders $\mathrm{H}$, et al. Proliferation characteristics in pediatric Hodgkin's lymphoma point to a cell cycle arrest in the G(1) phase. Mod Pathol 2005;18:1440-1447.

50 Druillennec S, Dorard C, Eychene A. Alternative splicing in oncogenic kinases: from physiological functions to cancer. J Nucleic Acids 2012;2012:639062.

51 Lee JM, Yoo JK, Yoo $\mathrm{H}$, et al. The novel miRNA hc-smR-S2-5 decrease the proliferation and migration of human lung cancer cells by targeting c-Met. Mol Cancer Res 2012;11:43-53.

52 Migliore C, Petrelli A, Ghiso E, et al. MicroRNAs impair MET-mediated invasive growth. Cancer Res 2008;68:10128-10136.

53 Jang KW, Lee JE, Kim SY, et al. The C-terminus of Hsp70-interacting protein promotes Met receptor degradation. J Thorac Oncol 2011;6:679-687.

54 Di Renzo MF, Olivero M, Giacomini A, et al. Overexpression and amplification of the met/HGF receptor gene during the progression of colorectal cancer. Clin Cancer Res 1995;1:147-154.

55 Di Renzo MF, Olivero M, Martone T, et al. Somatic mutations of the MET oncogene are selected during metastatic spread of human HNSC carcinomas. Oncogene 2000;19:1547-1555.

56 Peace BE, Hughes MJ, Degen SJ, et al. Point mutations and overexpression of Ron induce transformation, tumor formation, and metastasis. Oncogene 2001;20: 6142-6151.

57 Senchenko VN, Liu J, Loginov W, et al. Discovery of frequent homozygous deletions in chromosome 3p21.3 LUCA and AP20 regions in renal, lung and breast carcinomas. Oncogene 2004;23:5719-5728.

58 Birchmeier C, Birchmeier W, Gherardi E, et al. Met, metastasis, motility and more. Nat Rev Mol Cell Biol 2003;4:915-925.

59 Lai AZ, Abella JV, Park M. Crosstalk in Met receptor oncogenesis. Trends Cell Biol 2009;19:542-551.

60 Danilkovitch A, Donley S, Skeel A, et al. Two independent signaling pathways mediate the antiapoptotic action of macrophage-stimulating protein on epithelial cells. Mol Cell Biol 2000;20:2218-2227.

61 Follenzi A, Bakovic S, Gual P, et al. Cross-talk between the proto-oncogenes Met and Ron. Oncogene 2000;19:3041-3049.

62 Lee WY, Chen HH, Chow NH, et al. Prognostic significance of co-expression of RON and MET receptors in node-negative breast cancer patients. Clin Cancer Res 2005;11:2222-2228.

63 Lee CT, Chow NH, Su PF, et al. The prognostic significance of RON and MET receptor coexpression in patients with colorectal cancer. Dis Colon Rectum 2008;51:1268-1274.

64 Teofili L, Di Febo AL, Pierconti F, et al. Expression of the c-met proto-oncogene and its ligand, hepatocyte growth factor, in Hodgkin disease. Blood 2001;97: 1063-1069.

65 Rathore B, Kadin ME. Hodgkin's lymphoma therapy: past, present, and future. Expert Opin Pharmacother 2010;11:2891-2906.

Supplementary Information accompanies the paper on Modern Pathology website (http://www.nature.com/ modpathol) 